Patients and Methods 1880 individuals, from the 5 major garrison of the country, were double tested using two rapid tests. A Knowledge, Attitudes and Practice Survey (KAP) conducted. The results were analysed with STRATA and EPI INFO softwares.

Results Seroprevalence The seroprevalence rate among the whole armed forces was $\mathbf{1 . 1 8 \%}$ (IC95 0.89\%-1.55\%)

KAP survey The questioned individuals were 34 years for the average age, $55 \%$ were soldiers, $55 \%$ were been at Primary school, $97 \%$ quoted in first the AIDS like problem of public health in front of tuberculosis, the diabetes and malaria. 97\% quoted that in HIV prevention the most significant is fidelity, only $15 \%$ have carried out a HIV test in the last 12 months. $75 \%$ answered that the STD is contracted after a not protected sexual relation with an infected person. $90 \%$ think that the use of the condoms is prohibited by the religion. $90 \%$ of questioned people stated to consume Khat at least once per week. $65 \%$ think that sexual vagrancy is frequent under the negotiable instrument of Khat.

Conclusion The seroprevalence of the survey has shown a result of $1.18 \%$ which is lower than the awaited rate. Nevertheless we estimate that our methodology figuring close to the reality and it will be up to the Health Service of the armed forces to carry out prevention, information and care policy so that this rate does not grow in the future at best decrease in favour on an in-depth work.

\section{P3.345 ATTITUDES AND PRACTICES TOWARD PRE-MARITAL ROMANTIC/SEXUAL ACT AND CONDOM USE: A STUDY AMONG YOUNG INDIANS}

doi:10.1136/sextrans-2013-051184.0798

R Athimulam Kulasekaran. Annamalai University, Chidambaram, India

Background The initiation of sexual intercourse early in life is associated with an increased number of sex partners and a greater risk for STDs, HIV/AIDS.

Methods Data were drawn from Youth in India: Situation and Needs study. It is conducted by International Institute for Population Sciences, Mumbai and the Population Council, New Delhi and it focused on married and unmarried young women and unmarried young men $(50,848)$ aged $15-24$ during 2006-07.

Results There was a clear progression reported in physical intimacy and sexual experience with romantic/and/or other partners among the young in India. It is reported that 19 percent of young men and 9percent of women had been involved in a romantic partnership and significant proportion of young men (15percent) and young women (4percent) experienced pre-marital sex within romantic and/or other partnerships. Around 11percent of young men and 5 percent of young women had experienced first pre-marital sex before age 20. Young men tended to initiate pre-marital sex earlier than young women, moreover, about 47 percent of young men in rural areas, compared to 30 percent of those in urban areas, and 32 percent and 15 percent of young women, respectively, reported pre-marital sex with romantic partners. Around one-fourth of young men and little above one-fifth of young women had premarital sex with more than one partner and only 13percent of young men and 3 percent of young women used condoms in all premarital encounters. Unmarried youth were more likely than the married to report contraceptive use at first sex (30\% versus $23 \%$ among young men; $17 \%$ versus $8 \%$ among young women).

Conclusion This study underscore the fact that youth face numerous challenges while making transition to adulthood and these challenges should addressed by programmes/interventions at the youth, family and service delivery levels.

\section{P3.346 EVALUATION OF HIV, HBV, HCV, SYPHILIS AND RELATED RISK BEHAVIOUR PREVALENCE AMONG THE INJECTING DRUG USERS (IDU) IN THE CITY OF BARNAUL, RUSSIA}

doi:10.1136/sextrans-2013-051184.0799
N Vagaitseva, E Demyanenko. NGO "AntiAIDS-Siberia", Barnaul, Russian Federation

Background Different preventive programmes were implemented in Barnaul. Among them: condom and injecting equipment distribution, voluntary counselling and testing, social and medical support of the IDU and safe skills building. We investigated the difference in HIV, HIV, HCV, Syphilis and risk behaviour prevalence among the IDU programme clients (PC) covered by preventive programmes and the IDU who were not covered by preventive programmes (comparison group). Methods Integrated bio-behavioural survey with the use of "casecontrol" among the IDU was conducted in Barnaul, in 2011. 300 IDU were surveyed. Socio-behavioural, knowledge, HIV, HCV, HBV and Syphilis prevalence data were calculated with SPSS v.12.

Results The sample was: male (65\%), 24-45 years old (52\%), single (57\%), unemployed (79\%).

$94.7 \%$ of PC answered to knowledge questions correctly (5 key questions on HIV transmission and main preventive measures), in comparison group $-79.3 \%$.

$90 \%$ of PC did not use non-sterile needles and syringes for the last month, in comparison group - $84 \% .31 .3 \%$ of PC bought the syringes with ready -made solution, in comparison group $-48.0 \%$.

$25.3 \%$ of PC shared paraphernalia, in comparison group - $54.7 \%$.

$30.7 \%$ of PC used condom at the last sexual contact, in comparison group - $26.0 \%$

The results of testing: $3(2 \%)$ of $\mathrm{PC}$ are HIV positive, in comparison group - $31(20.7 \%)$

$2(1.3 \%)$ of $\mathrm{PC}$ have $\mathrm{HBV}$, in comparison group - $6(4 \%)$.

$121(80.7 \%)$ of PC have HCV, in comparison group - 130 (86.7\%).

7 (4.7\%) of PC have Syphilis, in comparison group - 35 (23.3\%).

Conclusions The IDU covered by the preventive programmes practise less risk sexual and injecting behaviour and prevalence of HIV, HBV, HCV, Syphilis among them is lower than among the IDU who never participated in preventive programmes.

\section{P3.347 ESTIMATED PREVALENCE OF T. VAGINALIS AND C. TRACHOMATIS AMONG YOUNG ADULTS: A LOCAL PERSPECTIVE}

doi:10.1136/sextrans-2013-051184.0800

\section{S M Rogers, MSSP Research Team. RTI, Washington, DC, United States}

Background Screening for C. trachomatis in the U.S. is recommended among women 25 years of age and younger. T. vaginalis (TV) is not a reportable disease, but has been associated with increased risk of other STIs. National prevalence data suggest that $\mathrm{TV}$ is common among African American women and increases with increasing age among women (Sutton et al. 2007). The local epidemiology of TV is not well understood.

Methods We compare population and subpopulation estimates of Ct and TV infections among a probability sample of 15 to 35-year olds residing in Baltimore, $\mathrm{MD}$, an urban area with high rates of STIs. The Monitoring STIs Survey Program (MSSP) collected survey data and urine specimens for STI testing from 2,120 participants in 2006-09.

Results The overall estimated prevalence of T. vaginalis was $7.5 \%$ (95\% CI 6.0, 9.0); infection was significantly higher among women $(11.8 \%)$ than men $(2.9 \%, \mathrm{OR}=5.1,95 \%$ CI $2.0,13.0)$. Over $75 \%$ of infections were asymptomatic. Among Black females, the estimated prevalence was $16.1 \%$ (95\% CI 12.8.19.5). Contrary to national estimates, rates of infection were significantly higher among subjects less than 26 years of age $(8.8 \%$ v $5.9 \%, p=0.04)$. In contrast, chlamydial infection was less prevalent overall, $3.9 \%$ (95\% CI 2.9, 5.2), and higher among men $(4.5 \%, 95 \%$ CI $2.8,6.9)$ than women $(3.4 \%$, $95 \%$ CI $2.4,4.8)$. Estimates of CT infection decreased with increasing age $(p<0.001)$. Nearly one-quarter of CT infections $(23.5 \%)$ were also positive for TV. 
Conclusion These data suggest a high burden of TV and co-infection with CT. Public health surveillance focusing on CT infection may consider routine screening of other STIs, including TV, in local populations to reduce STI morbidity.

\section{P3.348 MATERNAL AND CONGENITAL SYPHILIS REMAIN A CHALLENGE}

doi:10.1136/sextrans-2013-051184.0801

1.2D M S Magalhães, 'A Dias, II M P Calderon. 'Programa de Pós-graduação em Ginecologia, Obstetrícia e Mastologia da Faculdade de Medicina de Botuc, Botucatu, São Paulo/SP, Brazil, ' 2 Secretaria de Estado de Saúde do Distrito Federal, Brasília, Brazil

This descriptive study set the profile of pregnant with positive VDRL followed at public maternity hospitals in Federal District, Brazil, of the newborns children of mothers with syphilis that showed clinic signs of congenital disease and verified approach according with the recommendations of the Ministry of Health. The study verified the percentage of pregnant women who had access to prenatal care and were inadequately treated and the percentage of newborns with inadequate clinical management in accordance with the recommendations of the MS. We interviewed 67 pregnant as postpartum women who reported to the National System of Disease Notification, users of five public hospitals of the DF and consulted information from medical records and from prenatal care charts, when available. The information relating to the clinical and laboratory received to the children were supplemented with data from medical records, compulsory notification forms of syphilis, from prenatal care charts and the child health card, when available. Data were collected on sociodemographic, obstetric, information related to diagnosis, treatment of pregnant and puerperal women their partners and treatment of newborns into delivery. Only $41.8 \%$ patients were adequately treatment and main reason for inadequacy was the lack of treatment $(83.6 \%)$ or inadequate treatment of the partner $(88.1 \%)$. The study demonstrated the need for new treatment of the mother at the hospital for lack of documentation of the treatment in prenatal care. There was disagreement with the recommendations of the MS to follow-up of newborns with congenital syphilis in relation to radiographic study only $48 \%$ children carried out and lumbar pucture was required only $42 \%$. It was observed that $36 \%$ newborns not received any treatment. Our date demonstrates that the quality of prenatal care received by pregnant women is not sufficient to ensure control of congenital syphilis an reaching the goal of controlling the disease.

\section{P3.349 EPIDEMIOLOGICAL AND CLINICAL ASPECTS OF CONGENITAL SYPHILIS IN BELARUS}

doi:10.1136/sextrans-2013-051184.0802

0 Pankratov. Belarusian Medical Academy of Postgraduate Education, Minsk, Belarus

In the years 1996-2012 all together 4966 pregnant women infected with syphilis were registered in Belarus $(8.36 \%$ from the general number of the women with syphilis for this period - 59426 cases). Primary syphilis was diagnosed in $13.2 \%$ of patients, secondary syphilis - 30.5\%, latent early syphilis - $55.9 \%$, latent late syphilis $0.4 \%$. Disease has been revealed in I trimester of pregnancy in $56.7 \%$ of patients, II trimester $-23.2 \%$, III trimester $-17.5 \%$, after delivery $-2.6 \% .45 .1 \%$ of women have made abortion after disease revealing.

There are 127 children with congenital syphilis (CS) registered in 1994-2012 in Belarus in total, but last two years cases of CS have not been registered. Among women which have given birth to children with CS primary syphilis was not diagnosed, secondary syphilis $-26.7 \%$, latent early syphilis $-71.4 \%$, seroresistant syphilis $-1.9 \%$. Reinfection was registered in $8.5 \%$ of patients.
Early CS with symptoms (Table) has been revealed in $24(18.9 \%)$ of children, early latent CS - in $81.1 \%$. Cases of late CS have not been registered. Three children have died within the first month of a life.

Abstract P3.349 Table 1 Table-Clinical symptoms of manifest early CS in Belarus in 1994-2012 $(n=24)$

\begin{tabular}{lll}
\hline Symptom & Number of cases & $\%$ \\
\hline Pathology of bones: & 16 & 66.7 \\
osteochondritis II-III & 11 & 45.8 \\
periostitis & 1 & 4.2 \\
gummas of bones & 2 & 8.3 \\
pseudoparalysis of Parrot & & \\
Visceral pathology: & 17 & 70.8 \\
hepatosplenomegaly & 5 & 20.8 \\
hepatomegaly & 9 & 37.5 \\
congenital pneumonia & 2 & 8.3 \\
syphilitic nephritis & 2 & 8.3 \\
congenital heart disease & 1 & 4.2 \\
fibrosis of kidneys & 1 & 4.2 \\
fibrosis of adrenals & & \\
Pathology of nervous system: & 10 & 41.7 \\
encephalopathy & 3 & 12.5 \\
meningocephalitis & 2 & 8.3 \\
meningitis & 1 & 4.2 \\
hydrocephalus & & \\
Papules of skin and mucosas & 10 & 41.7 \\
Syphilitic rhinitis & 8 & 33.3 \\
Including with development of «saddle» nose & 1 & 4.2 \\
Syphilitic pemphigus & 5 & 20.8 \\
Roseola & 4 & 16.7 \\
Hochsinger's popular infiltration & 2 & 8.3 \\
Pathology of eyes: & 1 & 4.2 \\
syphilitic chorioretinitis & & 4.2 \\
syphilitic uveitis & & \\
\hline & & \\
& & \\
\hline
\end{tabular}

\section{P3.350 EVALUATION OF A COMPARATIVE WESTERN BLOT METHOD FOR EARLY POSTNATAL DIAGNOSIS OF CONGENITAL SYPHILIS}

doi:10.1136/sextrans-2013-051184.0803

'A Marangoni, 'P Nardini, , $\mathrm{C}$ Foschi, ${ }^{1} \mathrm{M}$ Compri, ${ }^{2} \mathrm{~A}$ Moroni, ${ }^{1} \mathrm{R}$ Cevenini. 'University of Bologna, Microbiology, DIMES, Bologna, Italy; ${ }^{2}$ S. Orsola Hospital, Microbiology, Bologna, Italy

Background Diagnosis of congenital syphilis (CS) remains difficult. Part of the problem arises because the standard serologic tests are not useful in newborns because IgG transfer across the placenta.

Since Western Blot technique allows the recognition of a specific response towards every single protein, it can be useful to compare IgG immunological profiles of mothers and babies at birth, in order to differentiate between passively transmitted maternal antibodies and antibodies synthesised by the infants.

\section{Methods}

Study group. Thirty infants born to syphilis seropositive mothers were enrolled for this study. At birth, routine serological tests were performed (ARCHITECT ${ }^{\circledR}$ Syphilis TP, Abbott; TPHA and RPR, Randox) on mother/child pairs' serum specimens.

"Home made WB". Treponema pallidum antigens, separated by SDS-PAGE, were blotted onto nitrocellulose sheets and incubated overnight with mother/child pairs' serum specimens.

Criteria for CS diagnosis were the following: presence of specific bands in the newborn's IgG WB strip different from those found on the corresponding maternal WB strip and/or recognition on IgM WB strip of at least 2 out the 4 following bands Tp47, TmpA, Tp17 and Tp15, including at least one with low molecular weight. 